\title{
Encircling silicone rod without drainage for retinal detachment with giant breaks
}

\author{
ABDEL-LATIF SIAM \\ Ophthalmology Department, Ain Shams University, Cairo, Egypt
}

Giant retinal breaks - those that extend $90^{\circ}$ or more round the circumference of the eyepresent difficulties to the surgeon and carry a relatively poor prognosis. Tears extending more than $180^{\circ}$, and particularly those with rolled edges or with the retinal flap bound down to the opposite side of the retina, require special skill and equipment. Massive preretinal retraction with shrinkage of the preretinal membranes is considered to be inoperable by most surgeons.

Cibis (1965) used an intravitreal cryoprobe to pull the posterior edge of the giant tear and incarcerate it in the sclero-choroidal wound. He preferred to inject air rather than silicone in such cases.

Schepens, Freeman, and Thompson (1965) and Schepens and Freeman (1967) have used the prone position, head exercises, and a specially designed rotating table to unfold retinal flaps. Freeman (1969), Freeman, Schepens, and Couvillion (1 970), and Couvillion, Freeman, and Schepens (1970) used intravitreal balloons to push and cryoprobes to pull the posterior retinal flap towards the other edge of the tear. The surgical procedure included choroidal incarceration, encircling silicone exoplants, and air injection.

Howard and Gaasterland (1970) described a useful technique based on the appreciation of the anatomical relationship between the periphery of the retina and the vitreous base. The detached vitreous base is sucked with a syringe through an opening in the pars plana under direct ophthalmoscopic control and is then incarcerated in the sclerotomy. A segmental buckle without drainage achieved a cure in the single case reported.

We have been trying for the last 5 years a simpler procedure to treat giant breaks which fulfil two requirements:

(i) They do not extend for more than $180^{\circ}$ round the circumference of the eye.

(ii) The retinal flap is not fixed to the opposite side of the retina, i.e. the posterior retinal flap is mobile.

During the last 5 years we have carried out an encircling procedure using a silicone rod $2 \mathrm{~mm}$. diameter without drainage of the subretinal fluid in twelve cases (Fig. I, overleaf). The retina was reposited and has remained so in nine cases, the most recent being of more than 6 months' duration.

\section{TECHNIQUE}

The conjunctiva is incised at the limbus and two radial cuts are made temporally and nasally as far back as the lateral and medial canthi. All four rectus muscles are dissected well back to obtain good exposure of the four quadrants of the sclera. The tear is localized at three points-both extremities and the 'estimated' posterior edge of the giant tear-and the sclera is marked with gentian violet 

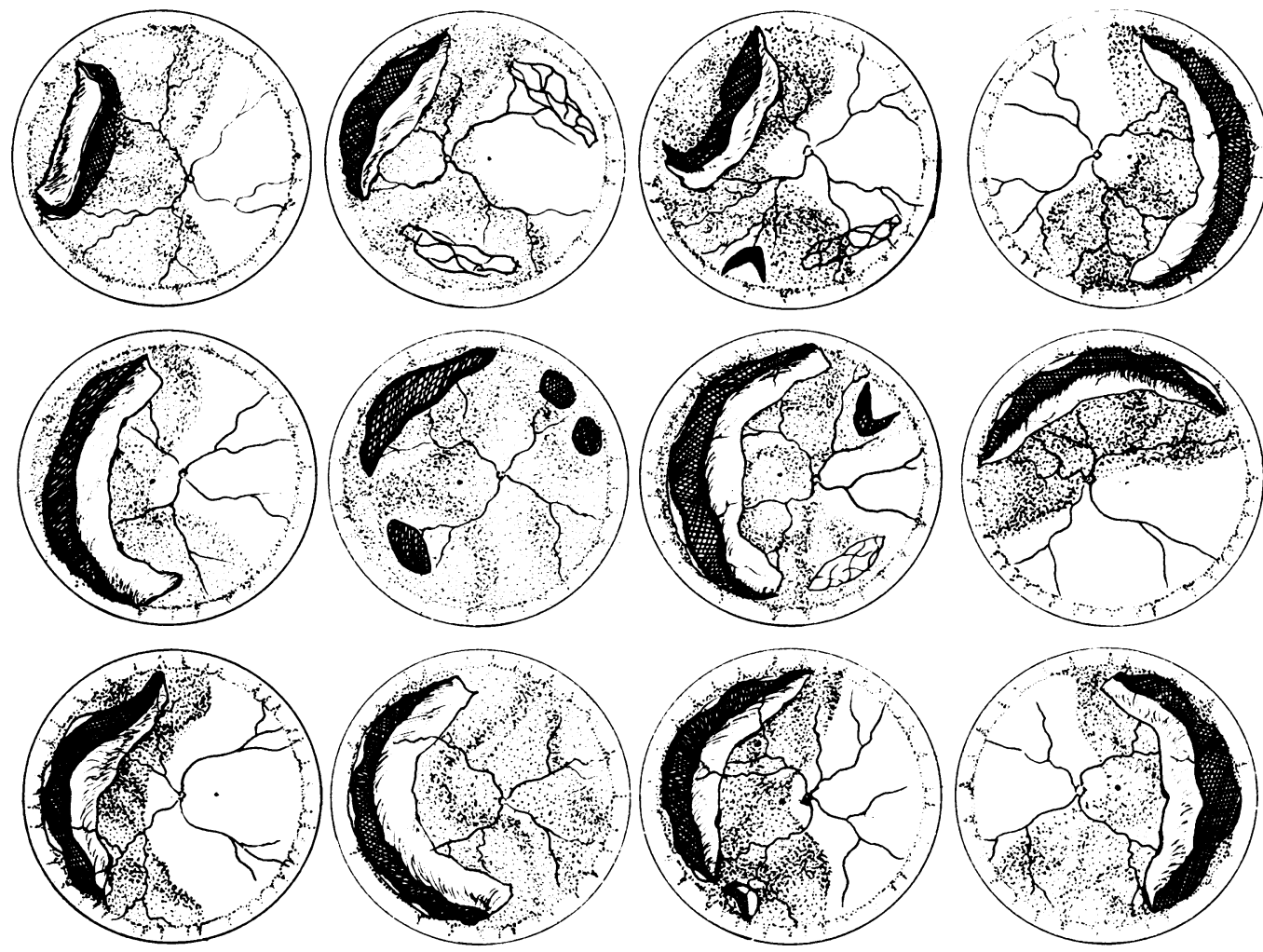

FIG. I Twelve cases of retinal detachment treated with encirclement without drainage of subretinal fuid

$2 \mathrm{~mm}$. beyond both extremities and $3 \mathrm{~mm}$. beyond the estimated posterior edge, since one cannot judge by the rolled-out posterior edge of the retinal break (Figs 2 and 3 ).

One should consider the localization in relation to the estimated posterior edge of the retina which is expected to come into contact with the forming buckle in the first postoperative days when the eye is becoming softer and the buckle becoming higher and higher (Fig. 3, interrupted line).

Cryoapplications (previously diathermy applications) are made from ora to ora in a double row and $3 \mathrm{~mm}$. beyond the estimated posterior edge of the giant break, and behind and beyond both extremities of the break (Fig. 3, small crosses). Other breaks are localized and treated in the usual way. Ordinary mattress sutures are placed using supramid $4 / 0$, taking care that the bites are $3 \mathrm{~mm}$. long and more than half the depth of the scleral thickness. The bites are taken $\mathrm{I} \cdot 5 \mathrm{~mm}$. beyond the treated area, i.e. about $4.5 \mathrm{~mm}$. between anterior and posterior bites. They should be closer together opposite the central part of the tear to ensure a high buckle behind the estimated posterior edge of the tear, and more anteriorly in the opposite quadrants but generally not far anterior to the equator. More recently, in the last three cases, we have been using the sutures described by Fradin (197I). Exact placement of sutures as regards their length and depth, and particularly in regions adjacent to vortex veins, is essential. At least some Fradin sutures are used when this seems advisable. Fradin sutures are certainly more time-consuming and more elaborate than ordinary mattress sutures, but they have the great advantage of less liability to accidental perforation. Other tears have to be taken into consideration and require a special arrangement of the sutures and the rod in their locality.

A silicone rod of $2 \mathrm{~mm}$. diameter is placed round the globe beneath the sutures, and both ends are pulled out until a buckle is visible. This has to be done very gradually and the pull sustained for a 


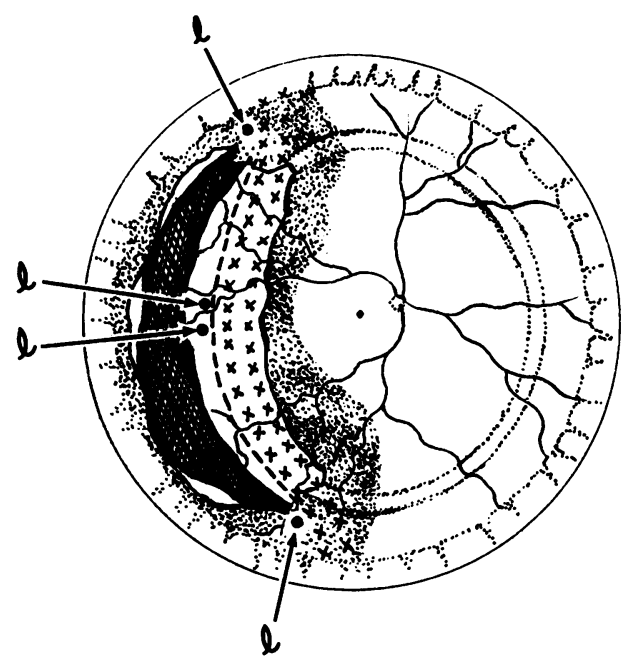

FIG. 2 Localization $(l)$ of retinal tear and cryoapplications ( $\mathrm{x} \times \mathbf{x} \mathbf{x})$

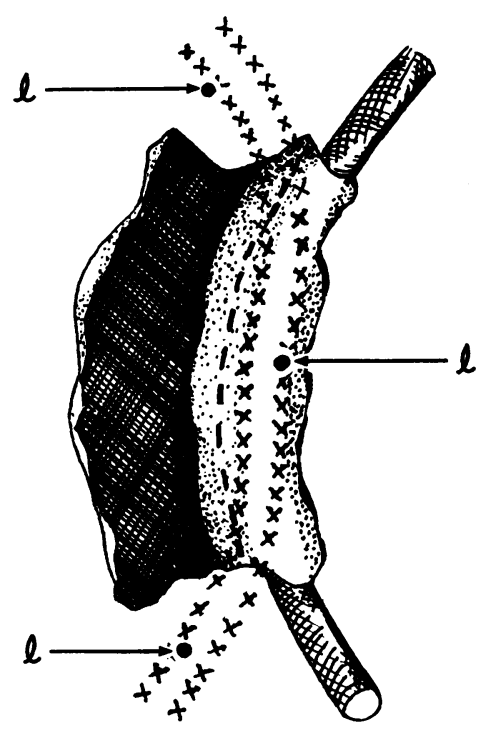

FIG. 3 Localization ( $l$ ) of retinal tear, with position of buckle $(---)$ and cryoapplications ( $\mathrm{x} \times \mathrm{x} \times \mathbf{x})$

few minutes to reduce the intraocular pressure: generally about 25 per cent. of the length of the rod has to be taken up. The rod is prevented from slipping by partial tightening of the most posterior and most anterior of the scleral sutures already placed. This procedure is also controlled by observation of the central retinal artery. The artery can remain closed for several minutes without any notable damage to retinal function. Both ends are fixed to the encircling part of the rod. The rest of the sutures are well tightened, starting with those opposite the estimated posterior edge of the retinal break. This process has to be very gradual and several minutes may elapse between two sutures. A constant digital pressure on the globe for a few minutes will reduce the intraocular pressure and allow the next suture to be tightened more effectively. Both ends of the silicone rod are brought under one of the mattress sutures opposite the giant tear (Fig. 4, overleaf).

If great patience is exercised while tightening the stitches, a paracentesis is hardly necessary at the end of this tedious step, whereupon a satisfactory buckle will form. It is astonishing how the rather discouraging fundus appearance will change in the postoperative days.

At the end of the operation the wound is irrigated with penicillin solution and the conjunctiva is closed with interrupted $6 /$ o black silk sutures.

\section{Discussion}

We first started this technique in 1966 when we operated on a medical student with a giant upper nasal break from 12 to 3 o'clock in the right eye (Fig. 4, overleaf).

The retinal flap and detached retina were extremely mobile and the eye was very soft. An encircling procedure was thought necessary and this was performed without evacuation of the subretinal fluid. Firm tension was just attained when all sutures were drawn up. The fundus appearance was not promising, with many radial folds along the buckle. This case, however, went very well in the following days; the folds started to flatten and the fluid absorbed gradually over the first 2 postoperative weeks. The retina was replaced and has remained so for 6 years. Although the posterior edge of the giant tear was everted, the retina adhered well to the circular buckle behind and beyond this everted edge (Figs 3 and 5 ). The radial folds became extremely narrow and flat 

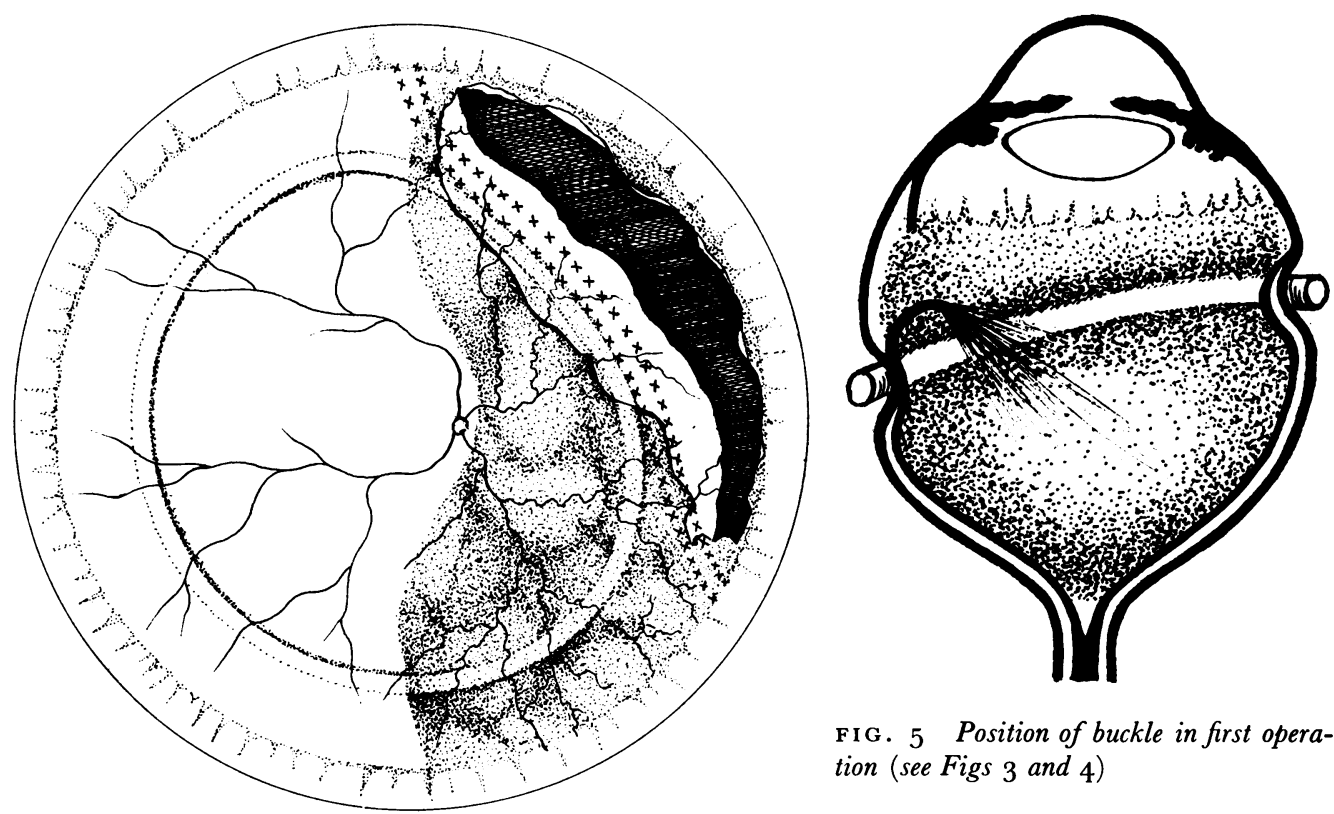

FIG. 5 Position of buckle in first operation (see Figs 3 and 4 )

FIG. 4 First operation, carried out in I966 (see Fig. 3)

in their peripheral ends where they disappeared in the scar on the circular buckle.

Impressed by the simplicity of the technique and encouraged by the unexpectedly good result, we applied this technique to our next twelve cases (Fig. I). The problem of the increased intraocular pressure has been dealt with in the description of the operative procedure. One very difficult complication which may arise is accidental perforation, which may happen with the most experienced surgeon. This is probably less likely with Fradin sutures. Cases in which accidental perforation occurred have been excluded from this report because they required intravitreal injections. In these cases, however, my experience has led me to favour air rather than silicone or other solutions. This has to be carefully and slowly injected to produce one large bubble rather than several small ones, to allow visualization. Slow injection is necessary, since the subretinal fluid, and sometimes the actual vitreous fluid, continue to drain. The tension may remain very soft if the air injection is done rapidly because of the continuous escape of fluid from the drainage site after the injection. In the manoeuvre of air injection one may find that one has actually replaced most of the vitreous and not merely supplemented it.

Other techniques which are too elaborate for the average busy retinal surgeon, and require much sophisticated equipment, also carry many risks. In most series the success rate is rarely better than $5^{\circ}$ per cent. but in our series nearly 75 per cent. were successful.

Cibis (1965) used intravitreal cryotherapy for cases like those in our series, namely cases of retinal detachment due to giant tears and rolled over retina. With the help of an intravitreal cryoprobe $1.5 \mathrm{~mm}$. in diameter, he pulled the retinal flap anteriorly and peripherally, and injected air after the retinal incarceration. Pulling on the retinal flap was criticised by Howard and Gaasterland (1970), who recognized that the vitreous base was firmly adherent to the peripheral retina and had found that in eye-bank eyes even gentle aspiration of detached peripheral retina into a needle-tip caused new retinal tears. Under direct vision, Howard and Gaasterland sucked the detached vitreous base with a 
syringe and were thus able to move the peripheral retina without tearing it. In the one case reported, the vitreous base was then incarcerated in the sclerotomy site over the pars plana, and the conventional buckling procedure was carried out without drainage.

Freeman and others (1970) and Couvillion and others (1970) used head exercises, a special operating table, and a human centrifuge to unfold the retinal flaps. Their surgical technique included choroidal incarceration, evacuation of the subretinal fluid, and air injection together with encircling procedures.

We do not feel it necessary to try to unfold retinal flaps when the procedure is so hazardous and requires so much skill and difficult intravitreal manoeuvres. Even if the flap is unfolded, one cannot be sure that the whole flap will adhere to its bed. I have seen cases operated on successfully in this manner, but great sectors of the central rolled-over retina remained rolled-out and loose in the vitreous. The retina adhered to the circular buckle well behind the flap as is achieved by our technique. It is surprising how the retina will go back during the prolonged proper positioning of the patient, especially when a high buckle is properly placed central to the rolled-out edge. The retinal flap was found to adhere to the circular buckle at the estimated posterior border of the tear. This is more likely when the retina is mobile, as pointed out by Scott (1970). In three of our cases it was obvious that the inverted retinal flap appeared to adhere to the buckle on its vitreous side.

\section{Addendum}

Since this paper was written, I have found that Okun (I g68), in a discussion with Davis, stated that he performed an episcleral encircling procedure without drainage of the subretinal fluid in a case of secondary retinal detachment due to diabetic retinopathy. He further stated that, encouraged by this result, he performed the operation on seventeen eyes with localized vitreous traction detachments. "In each instance, the detachment has either decreased in size or completely subsided despite the fact that no subretinal fluid was drained".

\section{References}

CIBIS, P. A. (1965) Trans. ophthal. Soc. U.K., 85, I I I

Couvillion, G. G., FREEMAN, H. M., and Schepens, G. L. (1970) Arch. Ophthal. (Chicago), 83, 715

FRADIN, s. (197 I) Ibid., 86, 58

FREeman, H. M. (1969) In "Symposium on the Retina and Retinal Surgery", p. i7I. Mosby, St.

Louis

59

HOWARD, R. O., and GAASTERLAND, D. E. (1970) Arch. Ophthal. (Chicago), 84, 312

OKUn, E. (1968) In "Symposium on Treatment of Diabetic Retinopathy", ed. M. F. Goldberg and

S. L. Fine, pp. 633,634 . U.S. Department of Health Education and Welfare

schepens, c. L., and freeman, H. м. (1967) Trans. Amer. Acad. Ophthal. Otolaryng., 71, 474

- - $\longrightarrow$, and тномpson, R. F. (1965) Arch. Ophthal. (Chicago), 73, $67 \mathrm{I}$

sCOTT, J. D. (1970) Trans. ophthal. Soc. U.K., 90, 57 